\title{
Laparoscopic Resection of a Huge Retrorectal Tumor
}

\author{
Han Deok Kwak, Jae Kyun Ju \\ Department of Surgery, Chonnam National University Hospital, Gwangju, Korea
}

Retrorectal space tumors are rare, and so are frequently unrecognized, misdiagnosed, and mistreated. A 57-year-old man visited the outpatient clinic with the chief complaints of thin stool and lower pelvic heaviness. A smooth, round huge palpable mass on the right posterolateral rectal wall was detected and pelvic computed tomography showed a 7.8-cm cystic lesion in the right retrorectal space. Laparoscopic procedures were initiated with perirectal dissection for rectal mobilization. After fixation of the peritoneum and tying the rectum for intracorporeal traction, the rectum was mobilized to identify the cyst. The cyst was removed using an endo-bag, with completion of cyst dissection. The final pathologic diagnosis was a tailgut cyst, or retrorectal cystic hamartoma without evidence of malignancy. The patient was discharged without any complications. The patient had no dyschezia or problems with bowel function. Laparoscopic resection is a safe and feasible method for surgical treatment, even for bulky retrorectal tumors, with an early recovery period.

Keywords: Retroperitoneal; Tailgut cyst; Tailgut cyst: Cystic harmatoma; Laparoscopy

\section{INTRODUCTION}

Retrorectal space tumors are rare, and so are frequently unrecognized, misdiagnosed, and mistreated. According to a case series from the Mayo Clinic [1], primary tumors in the retrorectal or presacral space are very rare, with an incidence of one in 40,000. Glasgow et al. [2] reported 34 primary retrorectal tumors in a span of 22 years. Tumors are most commonly benign in this area, but may also rarely be malignant. Thus definite radiologic diagnosis and precise surgical resection is required. Such tumors can be removed by various approaches. Surgery can be performed by the transperineal or abdominal approach, depending on the location of the lesion, as well as the preference of the surgeon, and a laparoscopic approach has also been recently reported. This report describes successful excision by laparoscopic surgery of a huge, symptomatic retrorectal tumor.

Received: April 30, 2018 - Accepted: July 31, 2018

Correspondence to: Jae Kyun Ju, M.D.

Department of Surgery, Chonnam National University Hospital,

42 Jaebong-ro, Dong-gu, Gwangju 61469, Korea

Tel: +82-62-220-6456, Fax: +82-62-227-1635

E-mail: jkju@chonnam.ac.kr

ORCID: https://orcid.org/0000-0003-1605-3310

(C) 2020 The Korean Society of Coloproctology

This is an open-access article distributed under the terms of the Creative Commons Attribution NonCommercial License (https://creativecommons.org/licenses/by-nc/4.0) which permits unrestricted noncommercial use, distribution, and reproduction in any medium, provided the original work is properly cited.

\section{CASE REPORT}

A 57-year-old man presented to the outpatient clinic with a complaint of thin stools since three months; he reported anal bleeding, outlet dysfunction constipation with incomplete emptying, and lower pelvic heaviness 1 week before his visit. The patient had undergone hemorrhoidectomy 8 years ago, and had no underlying diseases such as hypertension and diabetes. He had never undergone a colonoscopy before. Four centimeters proximal to the anal verge, at the 6 oclock position, a smooth, round huge palpable mass estimated to be more than $5 \mathrm{~cm}$ in diameter could be felt outside the rectum, accompanied by a small amount of bleeding on digital rectal exam. There was no visible tumor in the rectal mucosa, but mucosal modification was seen due to external compression at the same location on colonoscopy. Pelvic computed tomography $(\mathrm{CT})$ revealed a $7.8 \times 6.3 \times 7.2-\mathrm{cm}$ cystic lesion with thin, enhancing wall lining in the right retrorectal space. The tumor was located just below the level of the right seminal vesicle, superiorly abutting onto the right posterolateral wall of the rectum, pushing the rectum to the left (Fig. 1). On colonoscopy, there were no tumors in the mucosa, but external compression was noted, arising from the retrorectal space.

Preoperative bowel preparation was not done, and prophylactic intravenous 2nd generation cephalosporin was administered just before the operation. The patient was placed in the lithotomy position after induction of general anesthesia, to enable digital rectal exam during surgery. A $12-\mathrm{mm}$ trocar was inserted into the um- 
Volume 36, Number 1, 2020

Ann Coloproctol 2020;36(1):54-57

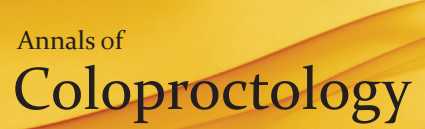

bilicus for the camera, and four 5-mm trocars were inserted into the right and left upper and lower quadrants, respectively. The small intestine was shifted upward with the Trendelenburg posi-
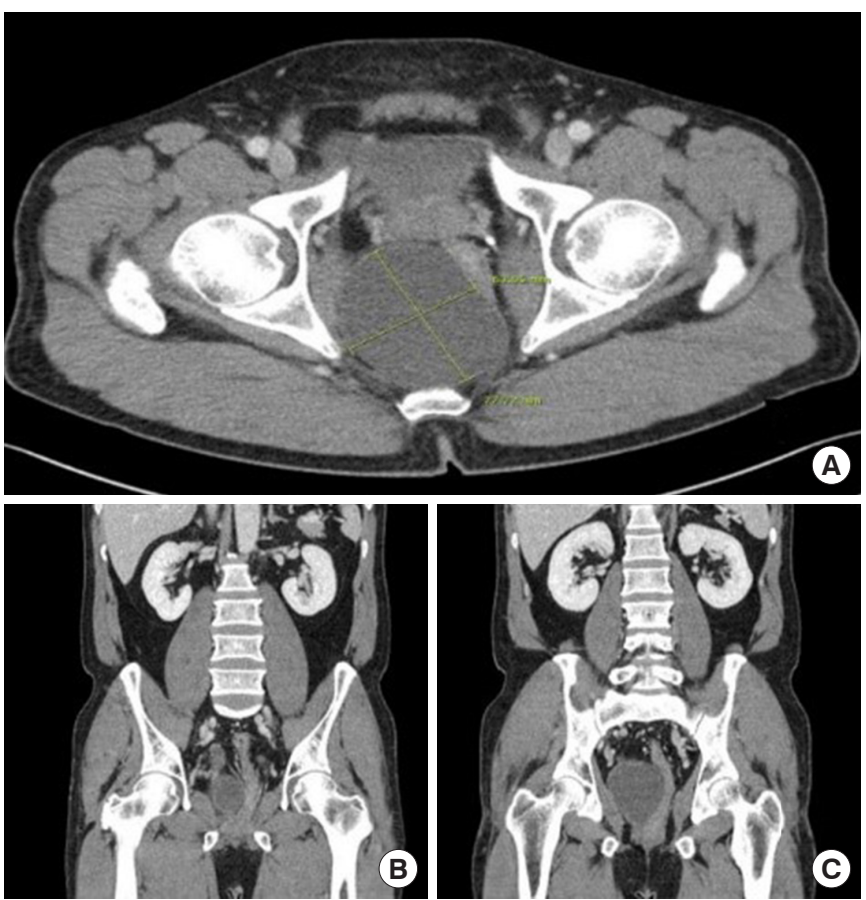

Fig. 1. (A) A $7.8 \times 6.3 \times 7.2$-cm-sized cystic lesion with thin, enhanced wall lining in the right retrorectal space. The tumor was located just below the level of the right seminal vesicle superiorly (B) abutting into the right posterolateral wall of the rectum, pushing the rectum to the left $(\mathrm{C})$.
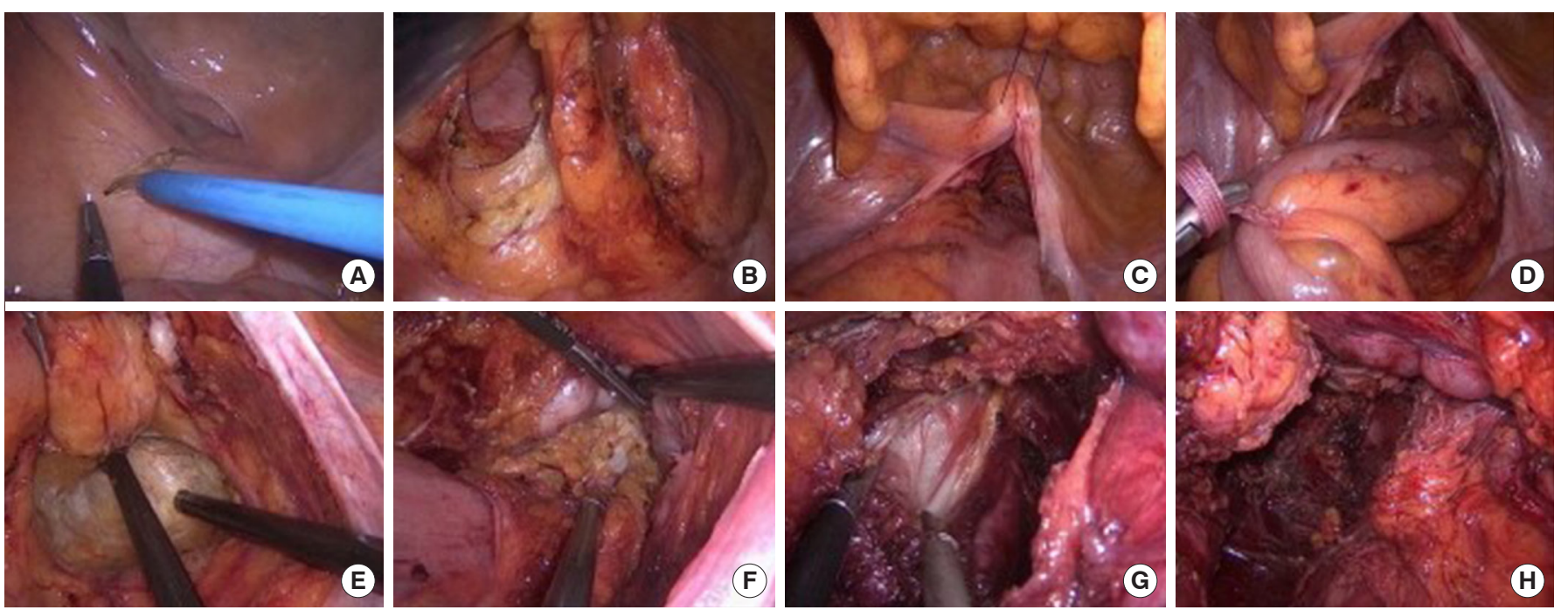

Fig. 2. Laparoscopic resection of the retrorectal tumor: perirectal dissection (A), rectal mobilization (B), peritoneal fixation with Prolene (C), tie-up of the rectum for intracorporeal traction (D), rectal mobilization (E), identification of the cyst (F), dissection of the cyst (G), and removal of the cyst $(\mathrm{H})$. 

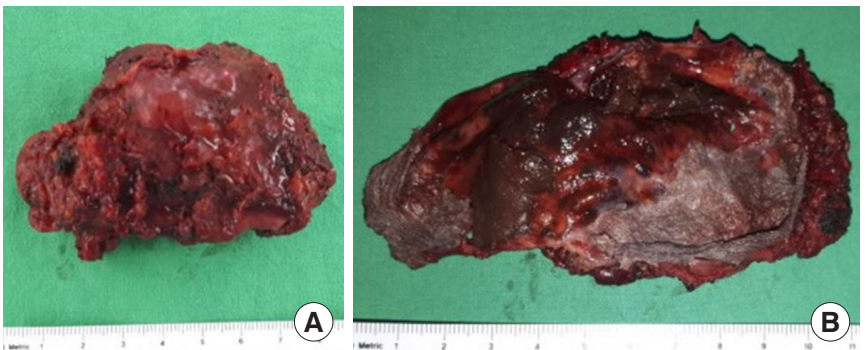

Fig. 3. (A) A $7.5 \times 5.0 \times 5.0$-cm-sized round shaped cyst compatible with tailgut cyst. (B) The interior of the tumor was filled with mudlike material.

The authors chose to operate laparoscopically as the lesion was slightly distant from the perineum and located in the narrow pelvis. Laparoscopy offered good visibility during the operation, effective hemostasis, and allowed for a short duration of hospitalization. The laparoscopic approach has gained popularity, owing to reduced surgical trauma and minimal vascular and neurological injuries, and is an excellent tool for perfect visualization of deep structures in the retrorectal space. In 2011, laparoscopic resection was reported for a retrorectal tailgut cyst at the level of S4 and the levator ani muscle measuring $4 \mathrm{~cm} \times 3.5 \mathrm{~cm} \times 3 \mathrm{~cm}$ [3]. Laparoscopic excision was described for nine retrorectal tumors from 2005 to 2011. Although 1 case required open conversion because of a huge tumor $>7 \mathrm{~cm}$, it was possible to reduce surgical trauma along with excellent visualization [4]. Surgeons must choose the surgical approach according to individual patient characteristics, considering the position of the tumor and its relationship with the pelvic structures. This report shows that laparoscopic resection is possible for huge retrorectal tumors through proper rectal mobilization.

As described earlier, a retrorectal tumor is a very rare condition, and the first case of this disease was described in 1885 [5]. According to a study that analyzed 53 cases in 1988, the disease is prevalent in women, with complaints of uncomfortable symptoms with accompanying inflammation in about half of the patients. One case showed poorly differentiated adenocarcinoma, but in most patients, the remnant of an embryonic tailgut was confirmed, which is different from teratoma [6]. A review article reported that various cystic lesions could occur in the retrorectal space, most of them being congenital. Those tumors are classified according to their origin and histopathologic features, but radiologic evidence is not diagnostic, and differential diagnosis can only be accomplished through histopathological analysis. Epidermoid or dermoid cysts arise from the ectoderm, while tailgut cysts and rectal duplication are thought to be from the remnants of the embryologic hindgut. Tailgut cysts are lined with various epithelia, unlike duplication cysts, which contain all components of the intestinal wall [7]. Retrorectal cystic hamartomas may in theory be classified as a teratoma, but may not have a dermal appendage, neural element, or derivatives such as cartilage or bone.
Many authors use the term retrorectal cystic hamartoma according to its anatomical location, and tailgut cyst depending on its developmental origin. This is, of course, supported only by pathological confirmation of the variety of epithelia [8].

In 2002, Ludwig and Reynolds [9] suggested that surgical extraction was necessary as cystic lesions may become spontaneously infected and increase the odds of recurrence, necessitating reoperative intervention. Further, a presacral mass may cause dystocia and prevent vaginal delivery, there may be an occult malignancy, or teratomas may degenerate and turn malignant. Removal can also improve the quality of life, and address cases with uncertain pathology [9]. Especially if it is suspected to be a malignant disease, preoperative diagnosis with a transsacral or transperineal biopsy of the tumor is essential to determine the necessity of neoadjuvant treatment, which may decrease local recurrence following surgery and avoid delays in instituting additional options [10]. When a tumor is diagnosed as malignant, multidisciplinary treatment includes colorectal surgeons, radiologists, and oncologists, who can contribute to successful treatment of the disease [2]. This case was an indication for surgical resection on account of uncomfortable symptoms and the large size of the tumor, which resulted in changes in bowel habit, although diagnostic imaging and colonoscopy showed minimal likelihood of malignant disease.

In conclusion, laparoscopic resection is a safe and feasible surgical treatment with early recovery, even for bulky retrorectal tumors.

\section{CONFLICT OF INTEREST}

No potential conflict of interest relevant to this article was reported.

\section{REFERENCES}

1. Jao SW, Beart RW Jr, Spencer RJ, Reiman HM, Ilstrup DM. Retrorectal tumors. Mayo Clinic experience, 1960-1979. Dis Colon Rectum 1985;28:644-52.

2. Glasgow SC, Birnbaum EH, Lowney JK, Fleshman JW, Kodner IJ, Mutch DG, et al. Retrorectal tumors: a diagnostic and therapeutic challenge. Dis Colon Rectum 2005;48:1581-7.

3. Lim SW, Huh JW, Kim YJ, Kim HR. Laparoscopy-assisted resection of tailgut cysts: report of a case. Case Rep Gastroenterol 2011; 5:22-7.

4. Nedelcu M, Andreica A, Skalli M, Pirlet I, Guillon F, Nocca D, et al. Laparoscopic approach for retrorectal tumors. Surg Endosc 2013;27:4177-83.

5. Caropreso PR, Wengert PA Jr, Milford HE. Tailgut cyst--a rare retrorectal tumor: report of a case and review. Dis Colon Rectum 1975;18:597-600.

6. Hjermstad BM, Helwig EB. Tailgut cysts. Report of 53 cases. Am J Clin Pathol 1988;89:139-47. 
7. Dahan H, Arrivé L, Wendum D, Docou le Pointe H, Djouhri H, Tubiana JM. Retrorectal developmental cysts in adults: clinical and radiologic-histopathologic review, differential diagnosis, and treatment. Radiographics 2001;21:575-84.

8. Prasad AR, Amin MB, Randolph TL, Lee CS, Ma CK. Retrorectal cystic hamartoma: report of 5 cases with malignancy arising in 2.
Arch Pathol Lab Med 2000;124:725-9.

9. Ludwig KA, Reynolds HL. Retrorectal tumors. Clin Colon Rectal Surg 2002;15:285-94.

10. Neale JA. Retrorectal tumors. Clin Colon Rectal Surg 2011;24: 149-60. 